\title{
CONVOLUTION ALGEBRAS ARISING FROM STURM-LIOUVILLE TRANSFORMS AND APPLICATIONS
}

\author{
JASON P. HUFFMAN and HENRY E. HEATHERLY
}

(Received 14 July 2000)

\begin{abstract}
A regular Sturm-Liouville eigenvalue problem gives rise to a related linear integral transform. Churchill has shown how such an integral transform yields, under certain circumstances, a generalized convolution operation. In this paper, we study the properties of convolution algebras arising in this fashion from a regular Sturm-Liouville problem. We give applications of these convolution algebras for solving certain differential and integral equations, and we outline an operational calculus for classes of such equations.
\end{abstract}

2000 Mathematics Subject Classification. 16S60, 44A40.

1. Introduction. Convolution operations serve as an interesting and fruitful link between analysis and algebra, for example, see [3, 4, 5, 7]. In this paper, we begin the study of a class of commutative, associative algebras that arise from regular SturmLiouville differential equations and their associated integral transforms and convolutions. The nexus is provided by results due to Churchill in the 1950's, but most readily referenced in [1, Chapter 10]. (Churchill's work was strictly in the differential equations-integral transform setting, with no reference to algebras.)

Consider the regular Sturm-Liouville system

$$
\begin{gathered}
\frac{1}{p}\left[\left(r u^{\prime}\right)^{\prime}-q u\right]+\lambda u=0, \\
\alpha_{1} u(a)+\alpha_{2} u^{\prime}(a)=0=\beta_{1} u(b)+\beta_{2} u^{\prime}(b) .
\end{gathered}
$$

Here $p$ and $r^{\prime}$ are continuous on the finite interval $[a, b], p$ and $r$ are positive on $[a, b]$, and $\alpha_{1}, \alpha_{2}, \beta_{1}, \beta_{2}$ are real numbers satisfying $\alpha_{1}^{2}+\alpha_{2}^{2} \neq 0 \neq \beta_{1}^{2}+\beta_{2}^{2}$. Recall that this system has an unbounded set of real eigenvalues, $\lambda_{1}<\lambda_{2}<\cdots$, each with a one-dimensional eigenspace, and corresponding eigenfunctions $\phi_{1}, \phi_{2}, \ldots$, [1, Chapter 10]. Churchill, [2, pages 325-343], made use of the regular Sturm-Liouville transform: $\mathbf{T}(F)=f$, where $f(n)=\int_{a}^{b} F(x) p(x) \phi_{n}(x) d x$, with $n \in \mathbb{N}$. (Here $\mathbb{N}$ is the set of all natural numbers and all the functions $F$ are real valued.) Taking $\Omega$ to be the subset of $C^{2}[a, b]$ of all functions for which the transform exists, we have that $\mathrm{T}$ is a linear operator from the space $\Omega$ into the space of all real-valued sequences.

The eigenfunctions $\phi_{1}, \phi_{2}, \ldots$, may be chosen so that they form an orthonormal set with respect to the inner product $\langle F \mid G\rangle=\int_{a}^{b} p(x) F(x) G(x) d x$. This orthonormal set has the property that if $F \in \Omega$ such that $\left\langle F \mid \phi_{n}\right\rangle=0$, for each $n \in \mathbb{N}$, then $F=0$. Consequently, $\mathbf{T}$ is injective. In order to obtain a useful convolution operation on $\Omega$ we further assume, following an idea introduced by Churchill, that $\mathrm{T}$ satisfies a weighted kernel product convolution property: there exists a positive sequence $\omega(n)$ such that 
for each $F, G \in \Omega, f \cdot g \cdot \omega \in \mathbf{T}(\Omega)$. (Here, “.” indicates componentwise multiplication of sequences.)

With the binary operation $\circledast$ defined via $f \circledast g=f \cdot g \cdot \omega$, the space $\mathrm{T}(\Omega)$ becomes an associative, commutative linear algebra over $\mathbb{R}$ (the real number field). Define convolution on $\Omega$ via $F * G=\mathbf{T}^{-1}(f \circledast g)$. Then $\mathbf{T}(F * G)=f \circledast g=\mathbf{T}(F) \circledast \mathbf{T}(G)$, both $\mathbf{T}$ and $\mathbf{T}^{-1}$ are $\mathbb{R}$-algebra isomorphisms, and the algebra $(\Omega,+, *)$ inherits the properties of the algebra $(\mathbf{T}(\Omega),+, \circledast)$. Note that the choice of $\omega$ may depend on finding a weighted kernel product convolution property that yields a concrete, computable convolution. (For the technical details on this process, from a strictly analytic point of view, see [2, pages 317-320].) It is clear that if $\omega(n) \neq 0$, for each $n \in \mathbb{N}$, then the algebra $(\Omega,+, *)$ has no nonzero nilpotent elements.

The subalgebras of $(\Omega,+, *)$ are exactly the subspaces of $\Omega$ which are closed under convolution. These subalgebras are the objects of study in this paper.

DEFINITION 1.1. Let $\Omega, \mathrm{T}$, and $*$ be as above and assume that $\omega(n) \neq 0$, for all $n \in \mathbb{N}$. A subalgebra of $(\Omega,+, *)$ which contains all of the eigenfunctions $\phi_{n}, n \in \mathbb{N}$, is called an SL-algebra.

It is well worth noting that most of the standard regular Sturm-Liouville transforms have the desired properties to yield SL-algebras, for example, the finite Fourier transforms and their modifications, (see [2, Chapter 11, Section 115]; several examples of concrete convolutions are given, as well as the weighted kernel product convolution properties from which they derive). And, although the conditions can be relaxed for individual problems yielding a convolution, we always take $\Omega \subseteq C^{2}[a, b]$ for simplicity and uniformity.

2. SL-algebras. It is easy to see (from the form of any $\mathbf{T}(F)$ ) that an SL-algebra is isomorphic to a subalgebra of the direct product of countably infinitely many copies of $\mathbb{R}$. Later, we will show that an SL-algebra is always isomorphic to a proper subalgebra of this direct product. In the sequel, $S$ will always denote an SL-algebra.

Because the algebraic structure of $\mathbf{T}\{S\}$ is often more tractable than that of the isomorphic algebra $S$, we make use throughout the paper of the a priori observation that all theorems proved for $\mathbf{T}\{S\}$ are entirely the same for $S$. For clarity, we adopt the convention that a capital letter $F$ represents a function in $S$, whereas a lowercase $f$ means an element of $\mathbf{T}\{S\}$.

We begin by introducing some classes of ideals generated by eigenfunctions in an SL-algebra $S$. For each $n \in \mathbb{N}$, define $K_{n}$ to be the ideal generated by the set $\left\{\phi_{j}: j \geq n\right\}$. Observe that $K_{1}$ is the ideal of $S$ generated by the set of all eigenfunctions. Next, let $E_{n}$ denote the principal ideal generated by the element $\phi_{1}+\phi_{2}+\cdots+\phi_{n} \in S$, for each $n \in \mathbb{N}$.

Proposition 2.1. Let $S$ be an SL-algebra. Then $S$ is non-Noetherian, non-Artinian, and has nonzero divisors of zero. In particular, $S$ does not have A.C.C. on principal ideals.

Proof. The desired chains of ideals are easier to see in the isomorphic image algebra. Observe that $\mathbf{T}\left\{\phi_{n}\right\}$ is the sequence with one in the $n$th component and 
zeros elsewhere. Then $E_{1} \subset E_{2} \subset \cdots \subset E_{n} \subset \cdots$ and $K_{1} \supset K_{2} \supset \cdots \supset K_{n} \supset \cdots$ are the chains required.

Since $\mathbf{T}\left\{\phi_{n}\right\} \circledast \mathbf{T}\left\{\phi_{m}\right\}$ is the zero sequence whenever $n \neq m$, we have $\phi_{n} * \phi_{m}=0$, and hence each eigenfunction is a (nonzero) zero divisor in $S$.

Let $\Sigma_{\mathbb{R}}$ be the direct sum of countably infinitely many copies of the algebra $\mathbb{R}$. This is the same as the set of all sequences which have finite supports. There exist ideals in $S$ which are isomorphic as $\mathbb{R}$-algebras to $\Sigma_{\mathbb{R}}$. For example, consider $\Psi: \Sigma_{\mathbb{R}} \rightarrow S$ defined via $\Psi\left(\left\{a_{n}\right\}\right)=\sum_{n=1}^{\infty} a_{n} \phi_{n} / \omega(n)$. Routine calculations show that $\Psi$ is an injective algebra homomorphism, and that $\operatorname{Im} \Psi=K_{1} \cong \Sigma_{\mathbb{R}}$. (Indeed, any ideal of $S$ generated by some infinite set of eigenfunctions will be isomorphic to $\Sigma_{\mathbb{R}}$.)

Also note that the ideals $E_{n}$ and $K_{n}$ have the following properties: $K_{n} \cong \Sigma_{\mathbb{R}}$ and $E_{n}$ is isomorphic to the direct sum of $n$ copies of $\mathbb{R}$, for each $n \in \mathbb{N}$.

The question arises whether an SL-algebra $S$ is isomorphic to $\Sigma_{\mathbb{R}}$. In general, the answer is negative, for there exist SL-algebras with elements whose images under $\mathbf{T}$ do not have finite support. For example, in an SL-algebra arising from the finite sine transform, we have $\mathbf{T}\{\pi-x\}=\{\pi / n\}_{n=1}^{\infty}$, which does not have finite support. In fact, the embedded image of $\Sigma_{\mathbb{R}}$ in $S$ need not be a maximal ideal. In SL-algebras arising from finite Fourier, cosine, and sine transforms, there exist elements whose images have infinite support (hence they do not lie in $K_{1}$ ) but whose product is zero. Thus $K_{1}$ is neither a prime nor a maximal ideal in this case.

Proposition 2.2. If I is an ideal of $S$ with $I \subseteq K_{1}$, then $I$ is a semiprime ideal of $S$.

Proof. Recall that $\omega(n) \neq 0$ for all $n \in \mathbb{N}$ and that $I \cong \mathbf{T}\{I\}$. Hence, if $f(n)^{2} \in \mathbf{T}\{I\}$ then we have that $\left(f(1)^{2} \omega(1), f(2)^{2} \omega(2), f(3)^{2} \omega(3), \ldots\right) \in \mathbf{T}\{I\}$. But $\mathbf{T}\{I\} \subseteq \mathbf{T}\left\{K_{1}\right\} \cong$ $\Sigma_{\mathbb{R}}$, and it is clear that any ideal of $\Sigma_{\mathbb{R}}$ must be semiprime. Thus, $\mathbf{T}\{I\}$ and hence $I$ must be semiprime ideals.

Proposition 2.3. An SL-algebra $S$ is a subdirect product of fields. Hence $\mathfrak{J}(S)=0$, where $\mathrm{J}$ is the Jacobson radical.

Proof. Recall that an SL-algebra $S$ is a subalgebra of the direct product of countably infinitely many copies of $\mathbb{R}$ and that $K_{1} \cong \Sigma_{\mathbb{R}}$. The mappings $\psi_{n}: S \rightarrow F_{n}$, where each $F_{n} \cong \mathbb{R}$ is a summand in $K_{1}$, defined via

$$
\psi_{n}\left[\left(a_{1}, a_{2}, \ldots\right)\right]=a_{n},
$$

are all surjective homomorphisms with kernels $M_{n}=\left\{F \in S:\left.\mathbf{T}\{F\}\right|_{n}=f(n)=0\right\}$. It is clear that $\bigcap M_{n}=0$ and that $S / M_{n} \cong \mathbb{R}$ for each $n$. Thus, each $M_{n}$ is a maximal ideal in $S$, and $S$ is a subdirect product of fields, which is necessarily Jacobson semisimple.

The maximal ideals of the form $M_{n}$ in the previous proof will be called standard maximal ideals. We will see that in several SL-algebras all maximal ideals are standard. It is of interest to note that for any eigenfunction $\phi_{n}$, we have $F-\phi_{n} * F \in M_{n}$, for all functions $F \in S$.

Although the Jacobson radical is zero in each SL-algebra, there always exist nonzero quasi-regular elements; that is, elements $F, G \in S$ such that $F+G+F * G=0$. 
For example, if $\omega_{1} \neq-1$, then routine calculations show that $\phi_{1}$ is quasi-regular. In fact, any linear combination of eigenfunctions, $\alpha_{1} \phi_{1}+\alpha_{2} \phi_{2}+\cdots+\alpha_{n} \phi_{n}$, will be quasi-regular provided that $\alpha_{j} \neq-1 / \omega_{j}$, for all $j=1,2, \ldots, n$.

3. The von Neumann regular radical. Although most of the classical radicals are zero in each SL-algebra, there is an important radical which is always nonzero. Recall that an element $a$ in an algebra $A$ is said to be von Neumann regular (VNR) if there exists $b \in A$ such that $a b a=a$. In this case, the element $b$ is called a pseudo-inverse of $a$ in $A$. (Note that this concept of regularity is distinct from that which determines a regular Sturm-Liouville system.) The unique largest ideal consisting entirely of elements of this type is an Amitsur-Kurosh radical (see [9, pages 192-195]), and this von Neumann regular radical, here denoted $\mathfrak{\lessgtr}(A)$, is nonzero for all SL-algebras. For several SL-algebras we characterize this radical $\mathfrak{\Im}(S)$.

Proposition 3.1. The VNR radical $\$(S)$ for any SL-algebra $S$ is nonzero. Furthermore, if $S$ has the property that $\{\omega(n)\}_{n=1}^{\infty}$ is constant or tends to zero as $n \rightarrow \infty$, then $\mathfrak{S}(S)=K_{1}$, the ideal generated by all of the eigenfunctions.

Proof. Let $S$ be an SL-algebra and recall that $K_{1} \cong \Sigma_{\mathbb{R}}$. It is routine to check that $\Sigma_{\mathbb{R}}$ is a von Neumann regular algebra, and hence $K_{1}$ is a nonzero VNR ideal in $S$. Thus, $\Im(S) \neq 0$.

Next, if $S$ has the property that $\{\omega(n)\}_{n=1}^{\infty}$ is constant or tends to zero as $n \rightarrow \infty$ (this is the case for many Sturm-Liouville transforms, including the finite Fourier, finite sine, finite cosine, etc.), then $\mathfrak{s}(S)$ is exactly the ideal $K_{1}$. To see this, observe that for any $F \in S \backslash K_{1}$ then $f(n) \neq 0$ for infinitely many $n$. Hence, the representation $f(n)=\mathbf{T}\left\{\sum_{n=1}^{\infty} f(n) \phi_{n}\right\}$ denotes an infinite sum. Therefore we must have that $\sum_{n=1}^{\infty} f(n)<\infty$, which implies $\{1 / f(n)\} \rightarrow \infty$, as $n \rightarrow \infty$. Furthermore, under the hypotheses, $\sum_{n=1}^{\infty} 1 / \omega(n)$ is a divergent series, and so the series $\sum_{n=1}^{\infty} 1 /\left(\omega(n)^{2} f(n)\right)$ is also divergent. A routine calculation shows that a pseudo-inverse of the element $F$ must be of the form $G=\sum_{n=1}^{\infty} 1 /\left(\omega(n)^{2} f(n)\right) \phi_{n}$, which clearly cannot exist. Hence, no element outside of $K_{1}$ may be VNR, and it follows that $K_{1}$ is the unique largest VNR ideal in $S$, that is, $K_{1}=\lessgtr(S)$.

Following the argument of the preceding proof, we see that no SL-algebra is isomorphic to the complete direct product of countably infinitely many copies of $\mathbb{R}$, for there are always sequences which cannot have inverse transforms. Indeed, whatever the weight sequence $\omega(n)$ is, there exist sequences $f(n)$ with the property that $\sum_{n=1}^{\infty} 1 /\left(\omega(n)^{2} f(n)\right)$ diverges. But because of the fact that $\$(S)$ always contains the ideal generated by the set of all eigenfunctions, $K_{1}$, we can say something about the "size" of $\mathfrak{\Im}(S)$ inside $S$.

Proposition 3.2. The VNR radical $\Im(S)$ is an essential ideal in $S$.

Proof. A nonzero ideal in an algebra $A$ is called essential if it has nonzero intersection with all other nonzero ideals of $A$. Let $0 \neq I$ be an ideal in $S$ and let $0 \neq F \in I$. We show that $\Im(S) \cap I \neq 0$. Since $F(x)=\sum_{n=1}^{\infty} f(n) \phi_{n}(x)$, where each $f(n) \in \mathbb{R}$, there exists some $j \in \mathbb{N}$ such that $f(j) \neq 0$ since $F$ is nonzero. But $f(j) \phi_{j} \in \mathfrak{s}(S)$ because $\mathfrak{S}(S)$ contains $K_{1}$. Thus, $0 \neq f(j) \phi_{j} \in \mathfrak{s}(S) \cap I$. 
In light of the previous proposition, we may say that an SL-algebra is, in some sense, "close" to being von Neumann regular.

Proposition 3.3. If all the maximal ideals in an SL-algebra $S$ are standard, that is, of the form $M_{n}$ for some $n \in \mathbb{N}$, then $S$ has no unity element. In particular, if $S$ has the property that $\omega(n)$ is constant or tends to zero as $n \rightarrow \infty$, then $S$ has no unity.

Proof. First, note that $\phi_{n} M_{n}=0$ and $\phi_{n} M \neq 0$, for each $n \in \mathbb{N}$, if $M$ is some nonstandard maximal ideal. This implies that the ideal $K_{1}$ is not contained in any standard maximal ideal and that $K_{1}$ must be contained by every nonstandard maximal ideal. Thus, if all the maximal ideals in $S$ are standard, then $K_{1}$ is not contained in any maximal ideal, and $S$ must have no unity. Next, if the condition on the weight function holds, then by Proposition 3.1, $\Im(S)=K_{1}$. Again, $K_{1}$ will not be contained in a maximal ideal, for if $M$ were one such, then $S / M$ would be a field, which contradicts the fact that $\mathfrak{\Im}(S)$ is the unique largest VNR ideal of $S$.

Propositions 3.1 and 3.3 give that an SL-algebra arising from a finite Fourier, sine, or cosine transform do not have a unity element. Furthermore, if all maximal ideals in an SL-algebra $S$ are standard, then there exist prime ideals which are not maximal. This is a consequence of the fact that $K_{1}$ is a semiprime ideal (hence the intersection of prime ideals) and that $K_{1}$ is not contained in $M_{n}$, for any $n \in \mathbb{N}$.

NoTE 3.4. It is worth mentioning that the sequence $\omega$ need not be bounded in order to satisfy a weighted kernel product convolution property. Indeed, Churchill has given an example of a convolution which yields an SL-algebra in which the weight is $\omega=2 n$, see [2, page 351].

Proposition 3.5. Let $S$ be an SL-algebra. If $S$ has unity, then $S$ is von Neumann regular.

Proof. We have shown that $\Im(S) \neq 0$. If $\Im(S) \neq S$, then $\Im(S)$ must be contained in a maximal ideal, say $M$. But $S / M$ is a field, which contradicts the fact that $\Im(S)$ is the unique largest VNR ideal of $S$. Hence, $\Im(S)=S$.

4. Applications. In this section, we describe several applications for SL-algebras in integral and differential equations. Existence theorems are given for certain classes of equations and, in some cases, uniqueness theorems as well. Note that although the existence of solutions for some of these equations is known, the approach here is unique: the algebraic properties of the convolution algebra yield the desired results directly. Thus, we circumvent the usual methods of proof while illustrating a beautiful connection between the algebra and the analysis.

First, consider the following proposition.

PROPOSITION 4.1. Let $S$ be the SL-algebra arising from the finite Fourier exponential transform and let $F \in S$ such that $F$ is a finite linear combination of eigenfunctions. Then the integral equation

$$
\int_{-\pi}^{\pi} \int_{-\pi}^{\pi} U(t) F(y-t) F(x-y) d t d y=F(x)
$$


with the unknown function $U$ has a solution in $S$ which is also a finite linear combination of eigenfunctions.

Proof. By hypotheses, we have $F \in K_{1}$, the ideal generated by all the eigenfunctions. But $K_{1} \subseteq \mathfrak{s}(S)$, and thus there exists a function $G \in K_{1}$ such that $F * G * F=F$. Writing out the convolution product gives the integral equation of the proposition, and thus $G$ is the desired result.

Proposition 4.1 is merely one example in a class of results. Note that an analogous proposition exists for each integral equation which corresponds to a convolution product of the form $F * G * F=F$ for an appropriate Sturm-Liouville transform. The existence of the solution function is an immediate consequence of the algebraic properties of $\mathfrak{s}(S)$ in $S$.

We have a similar class of results for convolution integral equations.

Proposition 4.2. Let $F \in S$, where $S$ is an SL-algebra arising from the finite Fourier exponential transform. If $K \in S$ has the property that $\mathbf{T}\{K\}=k(n) \neq-1 / \omega_{n}$ for each $n \in \mathbb{N}$, then the convolution integral equation

$$
U(x)+\int_{-\pi}^{\pi} K(x-y) U(y) d y=F(x)
$$

has a unique solution $U \in S$.

Proof. Under the hypotheses, $K$ is a quasi-regular element of the algebra $S$. Thus, there is a unique quasi-inverse, say $G \in S$, such that $K+G+K * G=0$. Now, it is clear by direct computation that the function $U=F+F * G$ is the desired solution. The uniqueness of $U$ follows from the uniqueness of $G$.

As before, there is an entire class of results for convolution integral equations of this type. Indeed for each convolution product resulting from a regular Sturm-Liouville transform, the existence and uniqueness of the solutions for these integral equations follows immediately from the algebraic properties of $S$. Note that there is no need for recourse to fixed-point theory or other analytic methods because of this connection with the algebra.

Perhaps the most intriguing application of the SL-algebras is the following connection to differential equations. The procedure of localization at a prime ideal is well known in commutative algebra. We recall some basic facts. The complement of a prime ideal $P$ in a commutative algebra $A$ is a multiplicatively closed subset which does not contain zero, that is, it is a denominator set. Then a ring of quotients, denoted $A_{P}$, can be formed consisting of terms $a / x$ where $a \in A$ and $x \in A \backslash P$. In an SL-algebra, each of the standard maximal ideals is prime and hence we may localize at any $M_{n}$. This allows the construction of a useful operational calculus which we illustrate with an example.

EXAMPLE 4.3. Consider the boundary value problem

$$
U^{\prime \prime}(x)-U(x)=F(x),
$$

where $F \in S$ and $U^{\prime}(0)=U^{\prime}(\pi)=0$. We may solve such a system as follows. In the finite cosine SL-algebra under the hypotheses, we have $\mathbf{T}\left\{G^{\prime \prime}\right\}=-n^{2} g(n)$, for any 
such $G \in S$. Thus, the system is equivalent to the following in the transform algebra $\mathbf{T}\{S\}$ :

$$
-n^{2} u(n)-u(n)=f(n) .
$$

We next consider this equation in the quotient algebra $S_{M_{1}}$. There exist elements, say $\sigma$ and $\delta$, in $S_{M_{1}}$ with the property $(\sigma-\delta) \circledast u(n)=-n^{2} u(n)-u(n)=f(n)$. The existence of $\sigma$ and is assured because of the fact that in this SL-algebra, the image of the function $A(x)=\pi / 12-(\pi-x)^{2} / 4 \pi$ under $\mathrm{T}$ is the sequence $-1 / n^{2}$. Thus, $A$ is in the complement of $M_{1}$ and hence is invertible in $S_{M_{1}}$. This gives that $A^{-1}=\sigma \in S_{M_{1}}$. Furthermore, $\delta$ is simply the unity element of $S_{M_{1}}$.

Now, we solve for $u$ in the above equation to get $u(n)=(\sigma-\delta)^{-1} \circledast f(n)$. Routine calculations show that the quotient $(\sigma-\delta)^{-1} \in S_{M_{1}}$ can be identified in the function algebra $S$, and in fact $(\sigma-\delta)^{-1}=B(x)$ where $B(x)=\mathbf{T}^{-1}\left\{-1 /\left(2 n^{2}+2\right)\right\}=$ $\cosh (\pi-x) /-2 \sinh \pi$. Thus, the solution of the boundary value problem is $U(x)=$ $B(x) * F(x)$, that is,

$$
U(x)=\frac{-1}{2 \sinh \pi} \int_{-\pi}^{\pi} \cosh (\pi-x+y) F(y) d y .
$$

It is well worth noting that the operational calculus presented above is particularly suited to differential equations containing even order derivatives of the unknown function. This is due to the operational property $T\left\{G^{\prime \prime}\right\}=-n^{2} g(n)$. The operational calculus illustrated in Example 4.3 may be of special interest to those solving applied problems in science and engineering with finite integral transforms. The interested reader is encouraged to see $[6,8]$ for more examples of problems in which the operational methods can be used.

Also, an interesting observation in the previous example is that the prime ideal $M_{n}$ used to create the quotient algebra actually depends on the differential equation being solved. In order for a certain function to be invertible in $S_{M_{n}}$, that function must not lie in the ideal $M_{n}$. Since any element $\gamma$ found by "factoring out" $u$ in $S_{M_{n}}$ (e.g., the element $(\sigma-\delta)$ above) must not be identically zero, then there always exists some $j \in \mathbb{N}$ such that $\gamma(j) \neq 0$ (often, there are many such indices $j$ ). This implies that neither $\gamma$ nor $\gamma^{-1}$ lie in $M_{j}$. Hence, the ideal $M_{j}$ is one which can be chosen for localization. Observe that the operational calculus developed here is not that developed by Mikusiński and has applicability to equations for which the Mikusiński operational calculus would be either awkward or of no use.

\section{REFERENCES}

[1] G. Birkhoff and G.-C. Rota, Ordinary Differential Equations, John Wiley \& Sons, New York, 1989. MR 90h:34001.

[2] R. V. Churchill, Operational Mathematics, 3rd ed., McGraw-Hill, New York, 1972. Zbl 227.44001.

[3] I. H. Dimovski, Convolutional Calculus, Kluwer Academic Publishers Group, Dordrecht, 1990. MR 91h:44001. Zbl 685.44006.

[4] H. E. Heatherly and J. P. Huffman, Noncommutative operational calculus, Proceedings of the 15th Annual Conference of Applied Mathematics (Edmond, OK, 1999), Southwest Texas State University, San Marcos, TX, 1999, pp. 11-18. MR 2001g:44012. Zbl 939.44001. 
[5] - Algebraic properties of the Mikusinski convolution algebra, Acta. Math. Hungar. 89(3) (2000), 179-187.

[6] N. A. Martynenko and L. M. Pustyl'nikov, Konechnye Integralnye Preobrazovaniya i ikh Primenenie k Issledovaniyu Sistem s Raspredelennymi Parametrami [Finite Integral Transformations and their Application to the Study of Systems with Distributed Parameters], Nauka, Moscow, 1986 (Russian). MR 88j:44001. Zbl 586.44001.

[7] T. W. Palmer, Banach Algebras and the General Theory of *-Algebras. Vol. I, Cambridge University Press, Cambridge, 1994. MR 95c:46002. Zbl 809.46052.

[8] G. Y. Popov, Elastic Stress Concentration Near Stamps, Cuts, Thin Inclusions and Supports, Nauka, Moscow, 1982 (Russian). Zbl 543.73017.

[9] F. A. Szász, Radicals of Rings, John Wiley \& Sons Ltd., Chichester, 1981. MR 84a:16012. Zbl 461.16009 .

Jason P. Huffman: Department of MATHematics, Computing, AND INFORMATION SCIENCES, JACKSONVILLE STATE UNIVERSITY, JACKSONVILle, AL 36265, USA

E-mail address: jhuffman@jsucc.jsu.edu

Henry E. Heatherly: Department of Mathematics, UniVersity of LOUisiana at LAFAYETTE, LAFAYETTE, LA 70504, USA 


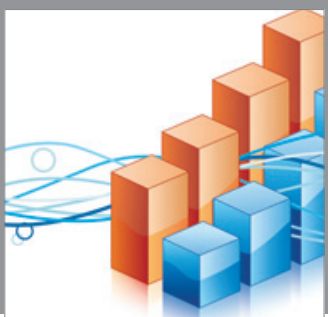

Advances in

Operations Research

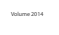

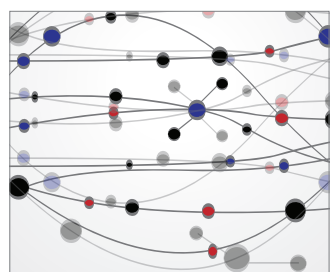

\section{The Scientific} World Journal
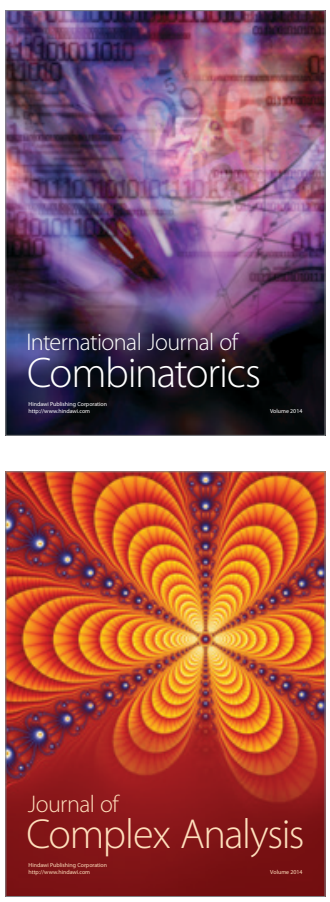

International Journal of

Mathematics and

Mathematical

Sciences
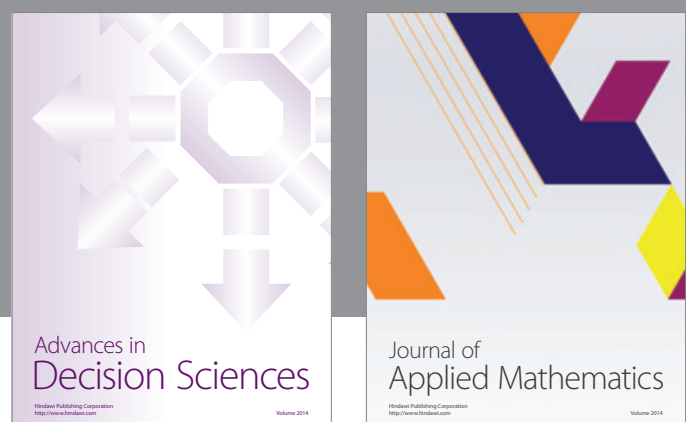

Journal of

Applied Mathematics
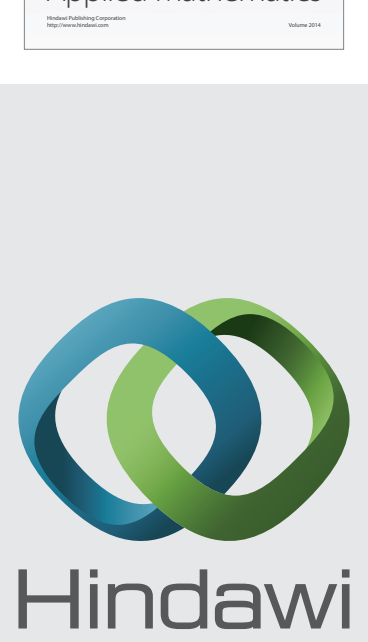

Submit your manuscripts at http://www.hindawi.com
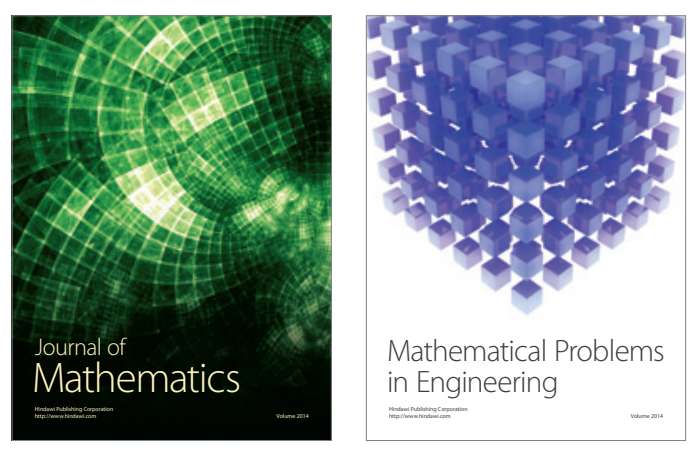

Mathematical Problems in Engineering
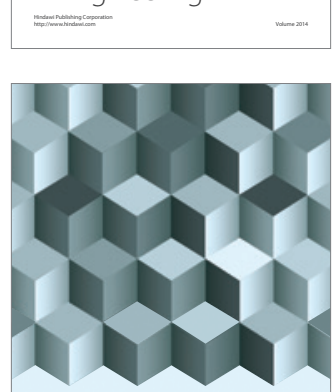

Journal of

Function Spaces
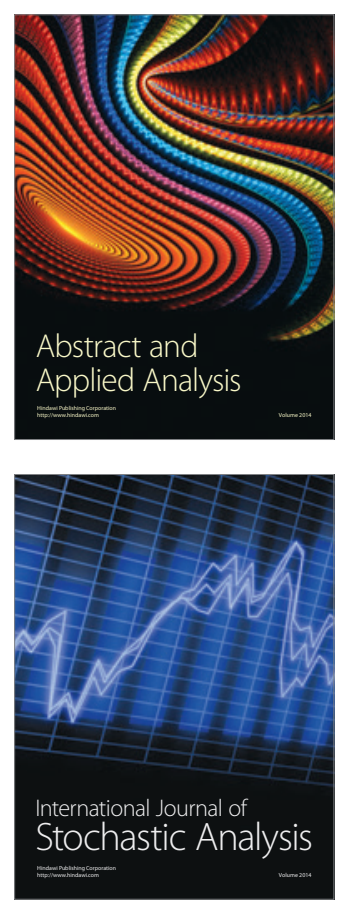

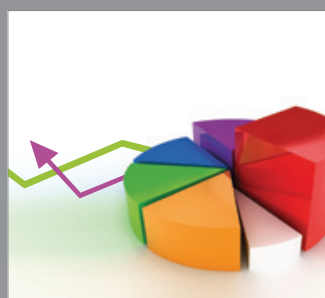

ournal of

Probability and Statistics

Promensencen
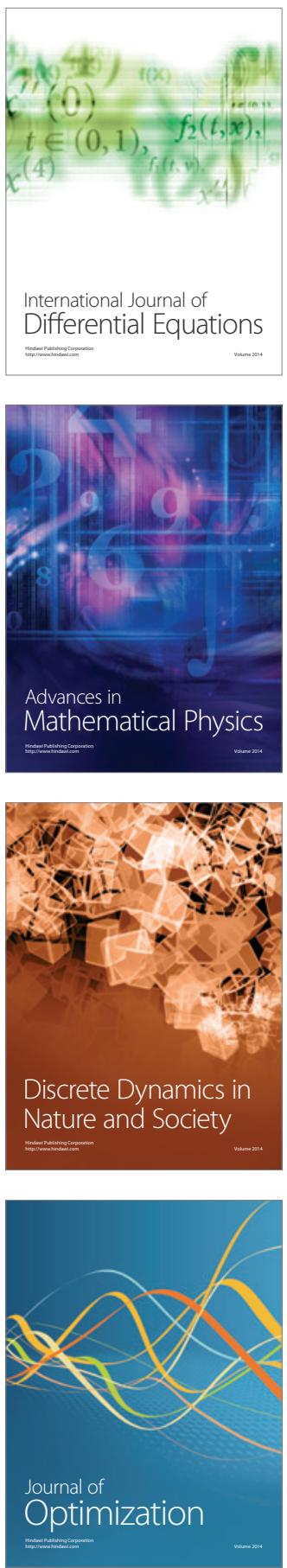